“C 2011 IEEE. Personal use of this material is permitted. Permission from IEEE must be obtained for all other uses, in any current or future media, including reprinting/republishing this material for advertising or promotional purposes, creating new collective works, for resale or redistribution to servers or lists, or reuse of any copyrighted component of this work in other works." 


\section{Intelligent Coordination Steering Control of Automated Guided Vehicle Vision-based}

\author{
Yuedong Zhan \\ Department of Automation \\ Kunming University of Science and Technology \\ Kunming 650051, China \\ ydzhan@163.com
}

\author{
Youguang Guo, JianGuo Zhu \\ Faculty of Engineering \\ University of Technology Sydney \\ NSW 2007, Australia \\ Youguang.Guo-1@uts.edu.au, Jianguo.Zhu@uts.edu.au
}

\begin{abstract}
In this paper, based on the neural network, fuzzy control and bang-bang control, an intelligent coordination control strategy for automated guided vehicle (AGV) steering system is presented. The dynamic steering model of distance error and orientation angle error for AGV is expressed. With least square method of system identification, the model of AGV is identified. Because a toy type of AGV is employed, its structure is simple, but AGV parameters are variable according to the operating conditions and environment, in order to improve the dynamic performances of $\mathrm{AGV}$, the intelligent coordinated control strategy is used to design the AGV controller in the AGV steering control system. Simulation and experimental results show the effectiveness of the proposed control strategy.
\end{abstract}

Keywords-automated guided vehicle (AGV); intelligent coordination control; neural network control; fuzzy control

\section{INTRODUCTION}

With the developments of Factory Automation (FA), Computer Integrated Manufacture System (CIMS) and the extensive application of the Flexible Manufacture System (FMS), and the Automatic Warehouse (AW), the field of the application and the level of the technology for Automatic Guided Vehicle (AGV), which link and adjust the discrete logistics system, have become the necessary tools for the automation transportations, loads and unloads, etc. Path tracking and steering control is the key technology among the researching fields of the $\mathrm{AGV}$, because it is very important to design an AGV steering control system, which has the following characteristics: smaller tracking error, quickly dynamic response, the adaptability to complicated circumstances, the robustness, and so on.

The AGVs were successfully developed and introduced in 1955 by the Basrrett Electric Company in America [1]. The AGVs have widely been used in industries since their introduction. The number of areas of application and variation in types has increased significantly. Meanwhile, the AGV technology developed rapidly in Europe, Japan, and Korea, because the sizes and structures of pallets for AGV had been standardized. At present, the AGVs can be not only used in inside, such as manufacturing, distribution, and transshipment, but also in outside environments, including the transportation areas [2].
AGV is a branch of mobile robot. With the progress of modern technology, the AGV will develop in the directions of intellectualization, autonomy-oriented, and industrialization. Moreover, there are a lot of advantages for AGV, such as unmanned-driven, good flexibility, stranger transportation ability, good safety, excellent environment protection, high utilization rate, and so on.

In the structures of AGV body, there are the three wheels type, four wheels type, and six wheels type. The more the wheels are, the stranger the loading capacity is, and better the stability is. The AGV body models are different with different the driving forms, but there are the motion models and dynamic models [3]. To the AGV steering control models, with the development of control theory, they are transfer function model, state-space model, autoregressive moving average model, fuzzy T-S model, neural network model, etc. In addition, in order to obtain and improve the performance of AGV control system in running environment and in case of many nonlinear constituents, they are not proper by design methods of controller using the traditional ones, such as PID control. However, it is suitable that the nonlinear control technique can act stably in the wide working range. To satisfy these conditions, there are many other methods, which are optimal control, self-tuning control, robust control, fuzzy control, neural network control, sliding mode control, reference model adaptive predictive control, genetic algorithm, and so on. On the other hand, the best control strategy must be that all kinds of methods mentioned above are integrated [4].

This paper firstly analyses the dynamic model for AGV steering control system based on a four wheels type of AGV in Section 2. Secondly, although the model and parameters can be identified, in order to obtain the reliability and the overall performances of a toy type of AGV, because of the effects of Coulomb's friction, error single amplitude limiting, backlash, and saturation nonlinear of the controller and PWM amplifier, an intelligent coordinating control (ICC) with the Bang-Band control, fuzzy control and neural network control has been employed for the AGV steering control to further enhance the robustness of the system in Section 3. Finally, in Section 4, the experimental setup and its results show that the proposal control strategy is of the feasibility, correctness and effectiveness. 


\section{DYNAMIC MODEL OF AGV STEERING CONTROL SYSTEM}

In this paper, the two-wheel difference speed steering control is used, assuming no slippage at the driving wheels, as shown in Fig. 1. Suppose the position error is $\Delta d(\mathrm{~m})$, orientation angle error is $\Delta \theta$ (degree), according to the body structure of $\mathrm{AGV}$ and the rigid translation principle, an AGV obeys the following set of dynamic equations with nonholonomic constraint [5].

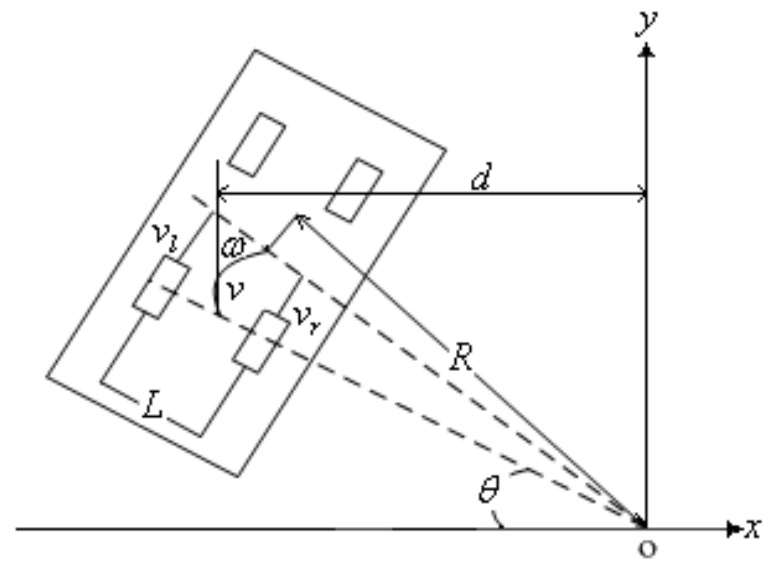

Figure 1. Definition of position erroe ( $\Delta \mathrm{d})$ and oritation error $(\Delta \theta)$ of AGV

$$
\begin{aligned}
& \frac{d \Delta \theta}{d t}=\frac{v_{l}+v_{r}}{2 R}=\frac{v_{l}-v_{r}}{L} \\
& \frac{d \Delta d}{d t}=\frac{v_{l}+v_{r}}{2} \cdot \sin \Delta \theta
\end{aligned}
$$

where $V_{l}$ is the left wheel speed $(\mathrm{m} / \mathrm{s}) ; V_{r}$ the right wheel speed $(\mathrm{m} / \mathrm{s}) ; L$ the distance between left wheel and right wheel (m); $R$ the turning radius (m), which is given by

$$
R=\frac{L}{2}\left[\frac{v_{l}+v_{r}}{v_{l}-v_{r}}\right]
$$

Let be the two wheels centre speed $V$ and speed difference of left wheel and right wheel $\Delta V$

$$
V=\left(v_{l}+v_{r}\right) / 2
$$

$$
\Delta V=v_{l}-v_{r}
$$

and that the orientation angle error is smaller, the state equations from (1) and (2) can be expressed as

$$
\left[\begin{array}{c}
\frac{d \Delta \theta}{d t} \\
\frac{d \Delta d}{d t}
\end{array}\right]=\left(\begin{array}{cc}
0 & 0 \\
V & 0
\end{array}\right)\left[\begin{array}{c}
\Delta \theta \\
\Delta d
\end{array}\right]+\left[\begin{array}{c}
1 / L \\
0
\end{array}\right] \Delta V
$$

Equation (6) shows that the speed difference of left wheel and right wheel $\Delta V$ is related to the position error $\Delta d$ and orientation angle error $\Delta \theta$. It can be treated as the state equations when the position error $\Delta d$ and orientation angle error $\Delta \theta$ are the state variables, the speed difference of left wheel and right wheel $\Delta V$ is the input variable. Therefore, the control of the speed difference of left wheel and right wheel $\Delta V$ can employed to adjust the position error $\Delta d$ and orientation angle error $\Delta \theta$.

In this paper, as a toy type of AGV is used as researching plant, and because the speed difference of left wheel and right wheel $\Delta V$ is not input value of AGV directly, the control input value of $\mathrm{AGV}$ is the time $\Delta T$ of switching value sent to the relay board in the PC, which the time $\Delta T$ is expressed as

$$
\Delta T=t_{l}-t_{r}
$$

where $t_{l}$ and $t_{r}$ are the switch conduction time of left wheel motor and right wheel motor (s).

To add the $\Delta T$ to the state equations as input value, the motion equations is needed to combine with the transfer function reflecting the dynamic performance of driving system, and the state-space model of AGV can be obtained, which is used to described the dynamic performance of AGV control process.

\section{INTELLIGENT COORDINATION CONTROL OF AGV}

In spite of the dynamic model of AGV has been deduced and identified, the nonlinear relationship between the position error $\Delta d$ and the orientation angle error $\Delta \theta$ is very complex. In addition, using the conventional PID control strategy, the control effects are worse. Because the practical deflection angle of the body centre of $\mathrm{AGV}$ is smaller than the rotation angle of wheel, when the orientation angle error $\Delta \theta$ is smaller, and the position error $\Delta d$ is smaller, the rotation control effect is better. However, although the orientation angle error $\Delta \theta$ is smaller, if the position error $\Delta d$ is larger, the rotation control effect is worse. 
Therefore, in order to enhance the control effects, and control abilities of self-adaptive, self-study, and robust of AGV, using the neural network to build the dynamic model of AGV, an intelligent coordination control strategy can be employed to control the whole process of AGV. Fig. 2 shows the structure diagram of intelligent coordination control for AGV.

In Fig. 2, the AGV control system consists of the information measurement, information processing, feature recognition, Bang-Bang control, fuzzy control, neural network control, intelligent coordinated controller, and so on. The key part is the intelligent coordinated controller, which can finish the coordination switching control of three kinds of control strategies [7].

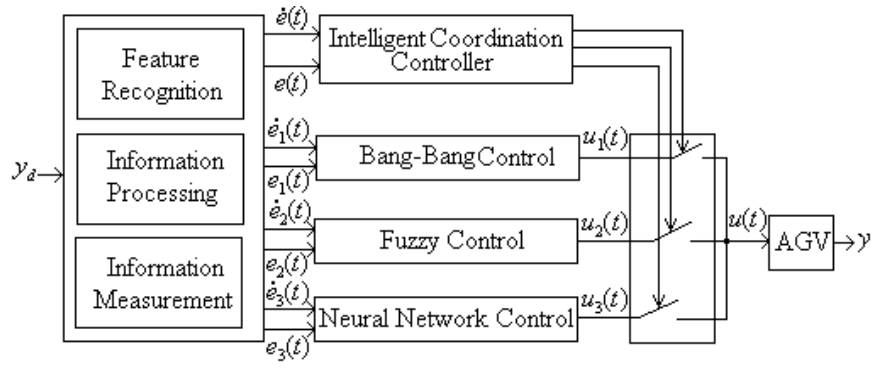

Figure 2. structure diagram of intelligent coordination control for AGV

\section{A. Design of Coordination Control Rules}

Based on the design thoughts mentioned above, according to expert experience, the coordination control rules are given as follows.

Rule 1: if $|e(t)| \geq E_{m}$ and $|\dot{e}(t)| \geq C_{m}$, then if $e(t) \geq E_{m}$, then $u(t)=u_{1}(t)=U_{\max }$; else if $e(t) \leq-E_{m}$, then $u(t)=u_{1}(t)=-U_{\max }$ (the Bang-Bang control) and (in this time, the neural network control is in the stage of learning).

Rule 2: if $E_{S} \leq|e(t)| \geq E_{m}$ and $C_{S} \leq|\dot{e}(t)| \geq C_{m}$, then $u(t)=u_{2}(t)$ (the fuzzy control) and (in this time, the neural network control is continuing to be in the stage of learning).

Rule 3: if $|e(t)| \leq E_{S}$ and $|\dot{e}(t)| \leq C_{S}$, then $u(t)=u_{3}(t)$ (the neural network control).

where $e(t)=y_{d}-y(t)$ is the position error and orientation angle error of the AGV; $\dot{e}(t)$ the change of error; $E_{m}$ and $E_{S}$ the threshold value of the error, and $E_{S}<E_{m} ; C_{m}$ and $C_{S}$ the threshold value of the change of error, and $C_{S}<C_{m}$. These parameters can be adjusted in the simulation and worksite.

\section{B. Fuzzy Control}

In this paper, based on the basis fuzzy control principle, a fuzzy controller of AGV path tracking has been designed. There are many different ways to use fuzzy controllers in closed-loop control applications. The most basic structure uses the sensor signals from the process as the input signals for the fuzzy controller and the outputs as the command values to drive the actuators for process control [8].

Fuzzy controller has two inputs variables $e(t), \dot{e}(t)$ and one output variable $u_{2}(t)$. In addition, there are various ways in which the observed input values can be used to identify which rules should be used to infer an appropriate fuzzy control action. The commonly used fuzzy inference methods are MAX-MIN fuzzy inference reasoning, MAX-PRODUCT inference reasoning, and SUM-PRODUCT fuzzy reasoning.

A basic and simple inference method, MAX-MIN fuzzy inference is employed in this paper. One of rules is expressed as

If $e(t)$ is $E_{i}$ and $\dot{e}(t)$ is $C_{j}$, then $u(t)=u_{2}(t)=u_{i j}, i=$ $1,2, \cdots, n ; j=1,2, \cdots, m$.

The membership of the inferred consequence $u(t)=u_{2}(t)$ is point-wise, which is given by

$$
\mu\left(u_{i}\right)=\underset{i, j}{\vee}\left(\left(\left(\mu\left(E_{i}\right) \wedge \mu\left(C_{j}\right)\right) \wedge \mu\left(u_{i j}\right)\right)\right.
$$

In this paper, the centre of area (COA) is selected for the defuzzification process in this paper, which is

$$
u_{2}(t)=\frac{\sum_{i=1}^{n} \mu\left(u_{i}\right) \cdot u_{i}}{\sum_{i=1}^{n} \mu\left(u_{i}\right)}
$$

\section{Neural Network Control}

The structure of designed intelligent neural network AGV control system consists of two types of neural network. The neural network controller (NNC) acts as the control network, which finishes the adjusting of PID parameters, and realizes the optimization of the performance of AGV; the neural network identifier (NNI) acts as the model identification of AGV plant, which finishes the modeling of AGV, and provides the learning signals. NNC and NNI can be firstly trained off-line according to the prior knowledge, and then made learning on-line when the AGV system is running in practice [9].

The traditional incremental digital PID control algorithm is defined as 


$$
\begin{aligned}
u(t)= & u(t-1)+K_{P} e(t)+K_{I}[e(t)-e(t-1)] \\
& +K_{D}[e(t)-2 e(t-1)+e(t-2)]
\end{aligned}
$$

where $K_{P}, K_{I}$, and $K_{D}$ are the coefficients of proportional, integral, and derivative, respectively, which denote the network weights, and let be $K=\left[K_{P}, K_{I}, K_{D}\right]$. Therefore, the online learning algorithm for NNC is given by

$$
\begin{aligned}
K_{i}(t+1)= & K_{i}(t)-\eta(t) \frac{\partial J_{C}\left(K_{i}\right)}{\partial K_{i}(t)} \\
& +\alpha\left[K_{i}(t)-K_{i}(t-1)\right]
\end{aligned}
$$

where $\eta(t)$ is the learning rate; $\alpha$ the smooth factor; $J_{C}(K)$ the performance index function learning on-line, using the steepest gradient descent rule, which is defined as

$$
J_{C}(K)=\frac{1}{2} \sum_{i=1}^{N}\left[e^{T}(t) Q e(t)+u^{T}(t) R u(t)\right]
$$

\section{EXPERIMENTAL SETUP AND RESULTS}

The performances of the proposed intelligent coordination control strategy of AGV steering system are tested by building an experimental setup, as shown in Fig. 3, whose key devices consists of a JK-1200 type of CCD camera, a DH-QP300 type of image acquisition card (wireless communication function), a multiple functions of digital I/O, A/D converter, D/A converter board (PLC-711B), a relay output board (PCLD-785B), a PC, and a toy type of AGV, and so on.

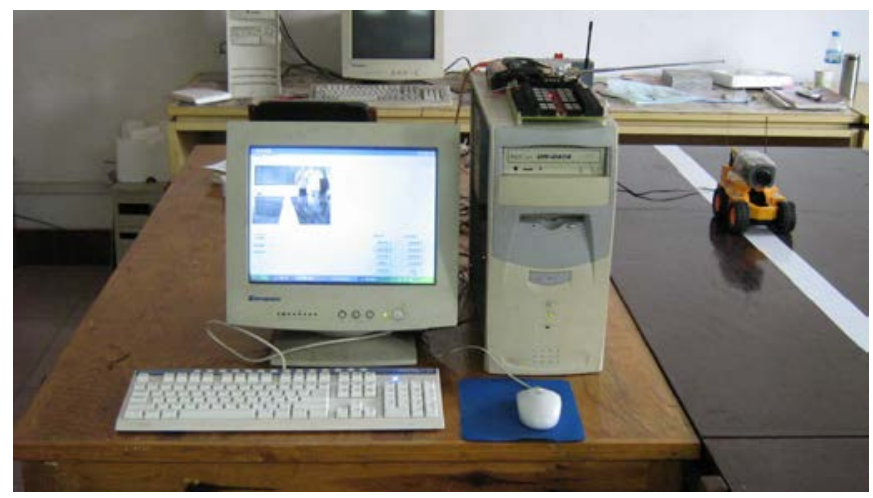

Figure 3. Experimental setup and environment for AGV

Fig. 4 reveals control performances of AGV, when the PID control strategy is used to control the AGV. The experimental results show that while AGV works in the PID control condition, it can run basically. However, in the deviation rectification of AGV steering process, the vibration of AGV is larger, and it is difficult to make the AGV run steadily.
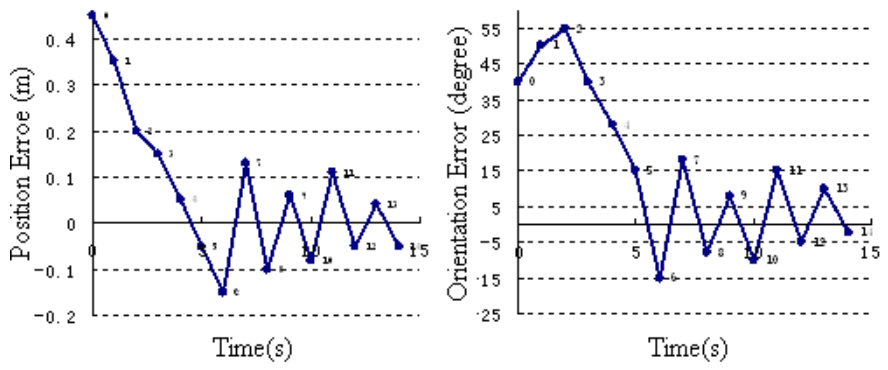

Figure 4. Control performances of AGV when the PID control strategy is used in AGV steering system

Fig. 5 shows the position error and orientation angle error of AGV when the intelligent coordination control strategy is applied in AGV steering system, which proves that the proposed control strategy is of the better features, such as fast dynamic response, short rise time, small overshoot, and keeping steadily running in smaller range of error. Its control effect is superior to the PID control.
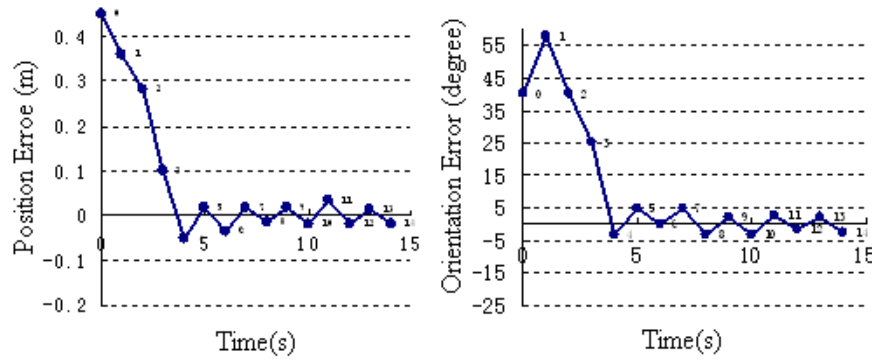

Figure 5. Control performances of intelligent coordination control strategy for AGV steering system

\section{CONCLUSION}

This paper investigates the intelligent coordination control strategy for obtaining the better performance of AGV at different operational conditions. The AGV steering system is a mobile robot system, which consists of a toy type of AGV, an image acquisition system, a data acquisition and control system, a PC computer, and a user control interface system. The intelligent coordinated controller has been designed to control the steering of AGV. The proposed intelligent coordinated controller includes the Bang-Bang controller, fuzzy controller, and neural network controller. The designed control system of AGV can on-line control the operating statues and parameters of AGV. The experimental results have proved that the proposed control scheme can work better than the conventional PID control, which is suitable for the nonlinear time-variable system, including the factors of the parameters changing, nonlinear features, and uncertain model of plant.

\section{REFERENCES}

[1] T. Muller, Automated Guided Vehicles. IFS Ltd., Springer-Verlag, Berlin, UK, 1983.

[2] F. A. Iris, "Survey of research in the design and control of automated guided vehicle systems," European Journal of Operational Research, vol.170(3), pp. 677-709, 2006. 
[3] F. G. Maughan, and H. J. Lewis, “AGV controlled FMS," International Journal of Production Research, vol 38(17), pp. 4445-4453, November 2000.

[4] W. Michiko, and F. Masashi, "Intelligent AGV driving toward an autonomous decentralized manufacturing system," Robotics and Computer-Integrated Manufacturing, vol. 17(1), pp. 57-64, Feb 2001.

[5] S. K. Tso, Y. H. Fun, and Y.P. Cheung, "Fuzzy-logic control for differential-wheel-drive AGVs using linear opto-sensor arrays," Proceedings of the 1996 IEEE International Conference on Robotics and Automation, Minneapolis, Minnesota, USA, pp. 2816-2821, April 1996.
[6] Y. S. Hou, System Identification and MATLAB Simulation. Science Press, Beijing, China, 2004.

[7] Y. N. Wang, Intelligent Control System. Hunan University Press, Changsha, China, 2006.

[8] E. M. Xi, Fuzzy Control Technology. University of Electronic Science and Technology of Xi'an Press, Xi'an, China, 2008.

[9] C. S. Jiang, C. Q. Wang, H. K. Wei, and M. Chen, Intelligent Control and Applications. Science Press, Beijing, China, 2007. 